This item was submitted to Loughborough's Research Repository by the author.

Items in Figshare are protected by copyright, with all rights reserved, unless otherwise indicated.

\title{
The application of simulation to the understanding of football flight
}

PLEASE CITE THE PUBLISHED VERSION

http://dx.doi.org/10.1177/1754337112444402

PUBLISHER

Sage Publications $@$ IMechE

VERSION

AM (Accepted Manuscript)

LICENCE

CC BY-NC-ND 4.0

\section{REPOSITORY RECORD}

Tuplin, Simon, Martin A. Passmore, David Rogers, Andy R. Harland, Tim Lucas, and Chris E. Holmes. 2012. "The Application of Simulation to the Understanding of Football Flight". figshare.

https://hdl.handle.net/2134/10036. 
This item was submitted to Loughborough's Institutional Repository (https://dspace.lboro.ac.uk/) by the author and is made available under the following Creative Commons Licence conditions.

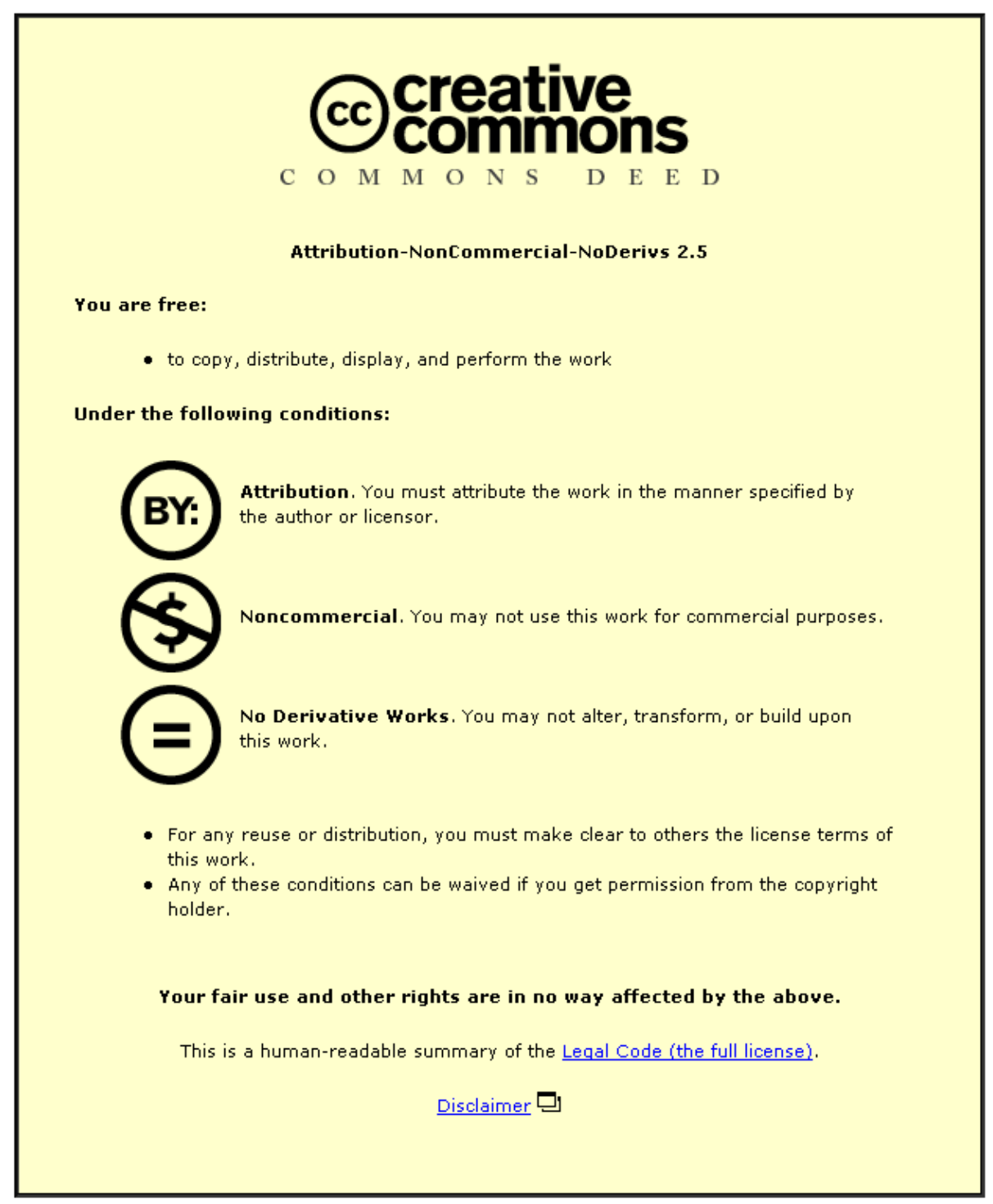

For the full text of this licence, please go to: http://creativecommons.org/licenses/by-nc-nd/2.5/ 


\title{
The Application of Simulation to the Understanding of Football Flight.
}

Simon Tuplin ${ }^{1}$, Martin Passmore ${ }^{1}$, David Rogers ${ }^{1}$, Andy Harland ${ }^{1}$, Tim Lucas $^{2}$, Chris Holmes $^{2}$

\begin{abstract}
This paper demonstrates the value of using a flight model in the analysis of the flight of a football and explores the complexity of the model required to produce useful results. Two specific aspects of the simulation are addressed; the need to include a model of spin decay and the requirement to include a full aerodynamic drag profile as a function of Reynolds number rather than a single indicative value. Both are aspects of the ball performance that are experimentally intensive to obtain.

The simulated flights show that the inclusion of spin degradation is important if flight validation is the objective but that it may be unnecessary in a comparative study. The simple analytical model of spin degradation is shown to over-estimate the reduction in lateral deviation when compared to experimentally acquired data. The experimental method is therefore preferred.

The analysis of the shape of the drag profile (drag coefficient against Reynolds number) is explored and it is shown from the simulated flights that post-critical coefficients of drag have the greatest effect on trajectories and an average drag value is sufficient for most modelled scenarios.
\end{abstract}

Keywords: Football, Aerodynamics, Flight trajectory, Spin Rate, Spin Decay, Reynolds Number

\section{Introduction}

The use of simulation is increasing throughout Engineering to provide insight into the performance of a product or system. It has particular value in the process of developing new products, where its application allows the characteristics of the product to be determined relatively early in the development cycle, thereby reducing development times and costs. Alternatively simulation may be used to gain an understanding of the importance of a particular parameter in the overall performance of the product. This paper uses a flight model, in combination with experimentally acquired aerodynamic data, in the analysis of the flight of a football. In this context the simulation provides the opportunity to explore the necessary inputs and complexity of the model required to produce useful results. To illustrate this the paper focuses on two specific aspects of the simulation, the need to include a model of spin decay and the requirement to include a full aerodynamic drag profile as a function of Reynolds number rather than a single indicative value. Both are aspects of the ball performance that are experimentally intensive to obtain.

It is usual, for ease of modelling, to assume that the initial spin rate imparted to the ball remains constant throughout a ball's flight, while in practice the spin rate decays as a consequence of the skin friction. To determine if it is necessary to include this additional complexity a simple analytical model of spin decay and the spin decay measured in the wind tunnel are implemented in the flight model. The effect on the flight in the two cases is compared with the assumption of a constant spin rate. The experimental method used to measure the spin decay is also described. It is often suggested ${ }^{1}$ that the drag coefficient as a function of Reynolds number, sometimes referred to as the drag profile, is important in determining the flight of the ball and that it is also potentially responsible for unpredictable behaviour. The use of a flight model makes it possible to study this effect directly by generating a number of different synthetic drag profiles and 
identifying the effect that these have on the flight of the ball. In this paper the Reynolds number at which transition occurs, the rate of transition and the level of the post transition drag coefficient are each studied.

\section{Background}

Aerodynamic effects play a crucial role in sports where the ball is given an initial impulse followed by a significant period of free flight. For example in football such situations occur in all parts of the play, including during passing, shots and in dead ball situations such as free kicks and corners. Typically the focus of the aerodynamic performance is on the period of free flight after the ball has been struck and before it comes into contact with the ground. During this period of free flight the aerodynamic force acting upon the ball can be separated into a drag component in line with the direction of motion and a lateral force perpendicular to the direction of travel. For the spinning ball the lateral force will also be perpendicular to the spin axis. The aerodynamic forces and pressure arising from the flow field surrounding a sphere are discussed at length in Achenbach's ${ }^{2,3,4}$ series of papers and an overview of sports ball aerodynamics, which has been studied extensively, can be found in Mehta ${ }^{5}$.

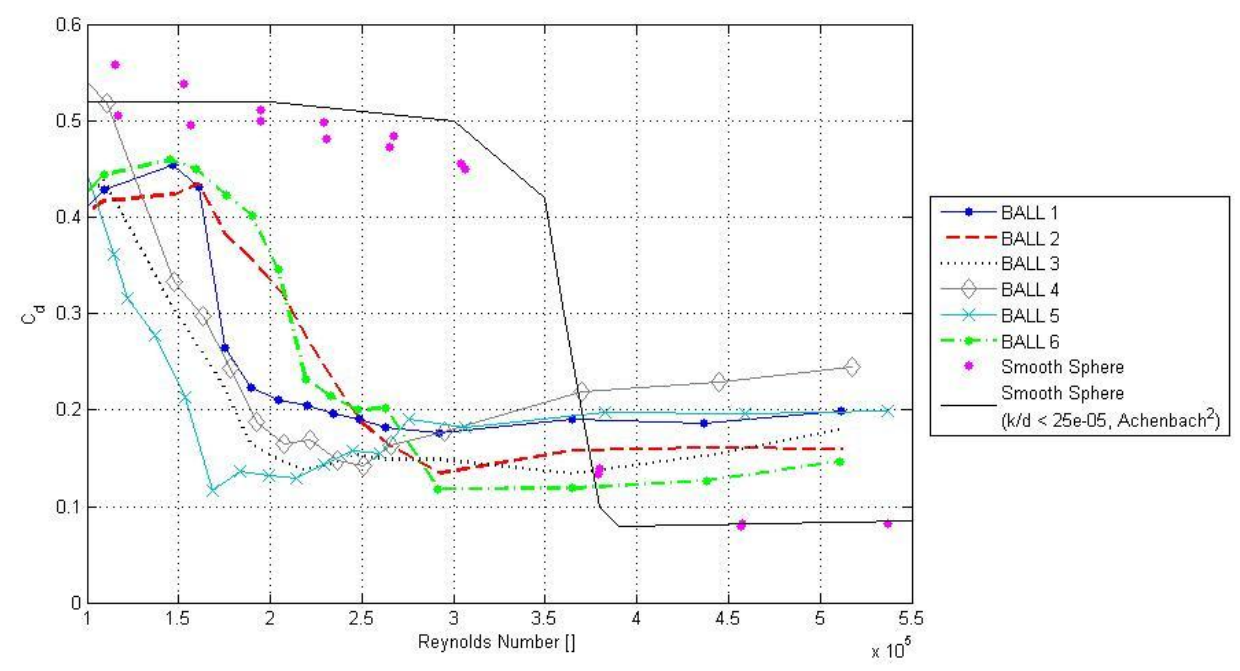

Figure 1 Drag coefficients against Reynolds number for a range of Footballs ${ }^{6}$

A comprehensive experimental study of the forces that are generated specifically on footballs can be found in Passmore ${ }^{6,7}$; drag and lateral force characteristics are reported for spinning and static balls and in the static case the lateral forces are shown to be a function of orientation.

Typical Drag coefficient against Reynolds number for a range of footballs is shown in figure 1 along with the results from Achenbach ${ }^{2}$. For the six real balls shown there are significant differences in the drag profiles. For example the lowest post critical drag is approximately 0.12 and the highest approximately 0.25 . Ball 2 has a relatively gentle transition from laminar to turbulent separation whereas for ball 5 it is relatively steep. 
The lateral coefficients for a spinning ball are shown in figure 2 against spin ratio ( ${ }^{\omega r} / U$ the spin ratio, is the ratio of ball surface velocity to free stream velocity) for a range of Reynolds numbers.

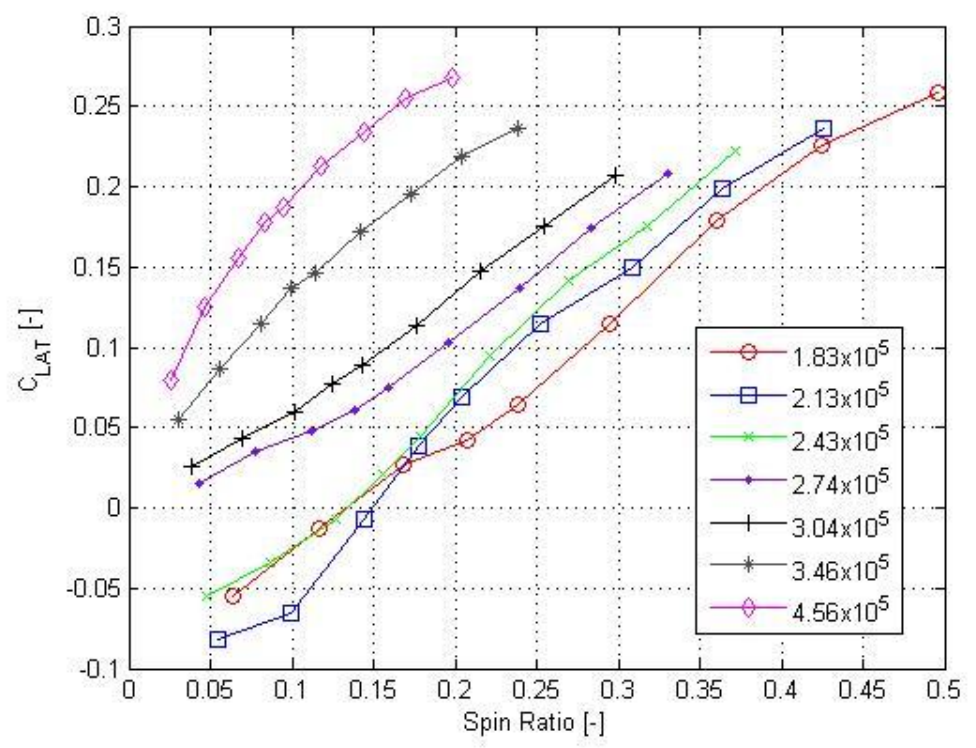

Figure 2 Lateral coefficients against Spin Ratio for a Footballs ${ }^{6}$

A similar study was undertaken by $\mathrm{Asai}^{8}$ who described in detail the flow field of a football for the non-rotating case, and also demonstrated that drag is dependent upon spin ratio for the spinning case. The wake structures were confirmed with flow visualisation. Earlier work by Carre $^{9}$ demonstrated that reverse Magnus effects are possible at low Reynolds numbers. The work was completed with a $1 / 3$ scale rapid prototype football $(66 \mathrm{~mm}$ diameter) and a 'mini' football (140mm diameter adidas Fevernova ${ }^{\mathrm{TM}}$ Replica). However with this approach the seams do not scale with the size of the ball. There was also a degree of uncertainty about the results reported for a spinning smooth sphere, being significantly different from other published data.

The use of flight models to understand the behaviour of a football is not new. The most comprehensive of those reported in the literature is that of Bray ${ }^{10}$ who developed a flight model in order to study the direct free kick. The aerodynamic data required in the model was inferred from a series of real kicks, captured using a video system to provide ball position and spin. The resulting data was fitted with the flight model to extract drag and lift coefficients. Goff ${ }^{11,12}$ published a method for extracting aerodynamic coefficients from flights captured with high speed cameras. The data shows reasonable agreement with a limited set of published windtunnel data. The spin imparted on the ball was for top spin only hence their analysis was limited to a single spin axis. However Goff ${ }^{12}$ states that although the analysis method was developed for three dimensional data, tracking in three dimensions was not possible with the equipment that they possessed. Passmore ${ }^{6,7}$ employs a model, based on that of Bray ${ }^{10}$, to make comparisons of the flight of a number of different balls but in this case the aerodynamic input is derived from wind tunnel tests. In addition Passmore ${ }^{6,7}$ explores the effects of low spin rates using a quasi- 
static implementation of the model; where data was acquired without spin through a range of yaw angles, and interpolation used in the flight model to calculate the forces for the appropriate onset orientation. Passmore ${ }^{6,7}$ then uses multiple simulated kicks to calculate the root mean square (RMS) deviation and hence characterise the ball performance. Cook $^{13}$ uses a model to evaluate the probability of success from a free kick and a corner kick, but doesn't discuss the fidelity of the model required. It is stated which solver works well but with no discussion of why and given that a high fixed value of drag coefficient $\left(C_{D}\right)$ is used the inherent error here would outweigh any effects of integration error.

At the engineering level it is important that we recognise the level of fidelity required from any simulation. An example of this is the recognition that during flight the spin imparted initially to the ball is dissipated by frictional effects that generate a resistive torque. Work by James ${ }^{14}$ established that spin decay exists and for a football (actual ball not specified) is approximately 3 times higher than tennis balls. However this study was limited to an onset velocity of $7 \mathrm{~ms}^{-1}$ and thus does not represent game appropriate velocities. Furthermore the ball will be in the subcritical regime with a laminar flow structure whose frictional properties are quite different. Consequently the range of spin parameters reported cannot be used to represent flight conditions.

Where aerodynamic data is obtained from wind tunnels a great deal of the published work $5,6,7,8,9$, begins with discussions of the non-rotating case; given that a ball is nearly always rotating during the game it must be questioned why an emphasis is placed upon the behaviour for the static case. It is true that this can be indicative of others responses, but cannot accurately describe any flight. The sensitivity of the aerodynamic loads to Reynolds number is also often quoted as being responsible for unusual flight behaviour ${ }^{1}$, though rarely with tangible evidence.

\section{Modelling Methods}

In the work reported here the flight model is based on that reported by Bray ${ }^{10}$ and implemented in Passmore et al ${ }^{6,7}$. The initial deformation of the ball, when struck, recovers sufficiently quickly that the ball can be assumed to be un-deformed throughout the flight ${ }^{15}$. It is clear that if trying to replicate an actual flight, the spin axis must be represented in three dimensions $10,11,12,13$ and as such this level of fidelity is included in the model; however for comparative work it is assumed to be vertical for simplicity and thus will generate a side force. The model is implemented using first-order backward differencing, where the time step $(t=0.001 \mathrm{~s})$ has been reduced until there is no significant change in the calculated flight path. More accurate or efficient integration schemes (c.f. Runge-Kutta) can be used but this is only considered appropriate for flight replication, as used in Goff ${ }^{11,12}$. The model can be used in two modes; a quasi-static mode, or spinning mode. In both cases the non-dimensional aerodynamic coefficients are obtained from an interpolation of the wind tunnel data for the particular ball type. For example in the spinning case the input data would be as is shown in figure 2 . The quasi-static mode is used to explore the effects of low levels of spin, so the interpolation uses data for the relevant coefficient against orientation angle measured with the ball static. This approach is not used in this paper. The aerodynamic data used in this paper is taken from that reported by Passmore ${ }^{5}$ unless stated otherwise.

The flight model can be used to predict a single flight, for a particular set of initial conditions, or used to generate a range of kicks across a representative range ${ }^{16}$ of initial launch conditions that 
can then be used to characterise the overall performance of the ball. Figure 3 shows the forces present on a ball during flight, with the direction of motion opposed by the drag force.

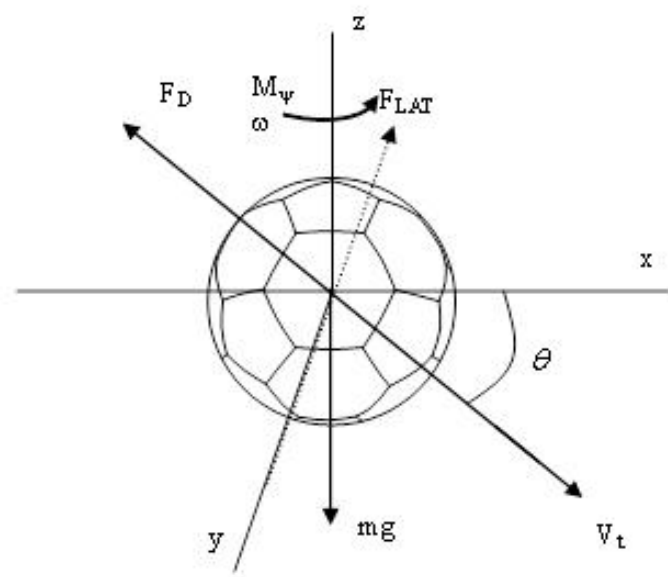

Figure 3 Forces on a ball during flight

From Newton's second law of motion the following equation describes, for a discrete time step $d t$, the balls flight:

$\overrightarrow{F_{D}}+\overrightarrow{F_{L A T}}+m \vec{g}=m \frac{d v_{t}}{d t}$

The drag and lateral forces $\left(\mathrm{F}_{\mathrm{D}}\right.$ and $\left.\mathrm{F}_{\mathrm{LAT}}\right)$ are calculated using equations 2 and 3 , which are populated from wind tunnel measurements contained within two dimensional look-up tables of the non-dimensional coefficients against Reynolds Number and spin rate.

$F_{L A T}=\frac{1}{2} \rho V_{t}^{2} A C_{L A T} \quad$ where $C_{L A T}=f(R e, \omega \mid \psi)$

$F_{D}=\frac{1}{2} \rho V_{t}^{2} A C_{D} \quad$ where $C_{D}=f(R e, \omega \mid \psi)$

And $\quad R e=\frac{\rho v d}{\mu}$

Given that the ball has the initial conditions $V_{t}, \theta, \omega, \psi$, [Launch Velocity, Launch Angle, Spin Rate, Yaw Rotation of ball)] the forces are calculated and orientated in Euclidean space with $\Theta, \phi, \varphi$,relating to Pitch, Roll and Yaw of the spin axis. For the spinning ball $F_{D}$ and $F_{L A T}$ are functions of both Reynolds number and spin rate. The use of the non-dimensional parameter of spin ratio is unhelpful for understanding the aerodynamic characteristics; as it is demonstrated in Passmore ${ }^{6}$ that the forces are not solely dependent on this ratio. For the low spin condition where quasi-steady conditions are assumed they are a function of Reynolds number and orientation angle. The cross over between low spin where the orientation is important and spinning ball where the Magnus force is dominant is unclear; for the purposes of this paper 1 $\mathrm{rad} / \mathrm{s}(\sim 10 \mathrm{rpm})$ is chosen. The lateral force acts normal to the plane containing the instantaneous velocity and spin axis. In the absence of actual measured flight data that precisely orientates the spin axis, it is a reasonable approach to keep the spin axis vertical. The translation of the ball, $s$, is given by:

$s=\iint_{t_{1}}^{t_{2}} \frac{\vec{F}}{m} d t$ 
The aerodynamic forces act through the centre of pressure which occurs at a distance of $\mathrm{x}_{\mathrm{cp}}$ from the origin of the ball; calculated from equation 6 and illustrated in figure 4.

$$
x_{c p}={ }^{M}{ } / F_{L A T}
$$

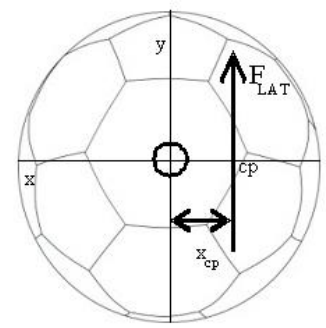

Figure 4 Centre of Pressure during flight

Consequently the angular velocity of the ball is a function of the yaw moment and is assumed to be applied normal to the spin axis. The angular velocity of the ball due to yaw moment is given by equation 7 and modifies the instantaneous angular velocity appropriately.

$\omega_{\psi}=f\left(M_{\psi}\right)=\frac{M_{\psi}}{I} \quad$ where $I \cong 2 / 3 m r^{2}$

The flight model can, if required, be extended to include spin rate degradation. Two approaches are taken in this work; spin decay rate from experimental tests can be invoked directly or, as described here, a model of the surface properties can be used. The ball surface is thus represented as two flat plates, one for the high pressure side of the ball and one for the low pressure side, see figure 5 , and summation across this surface allows the total frictional force due to the imparted spin to be estimated.

$F_{T}=1 / 2 \rho U_{T}^{2} C_{F} S_{B}$

The reference area $S_{B}$ is that of the ball surface approximated to uniform sphere $\left(4 \pi r^{2}\right)$. The tangential velocity at the ball surface, $\mathrm{U}_{\mathrm{T}}$, is estimated by equation 9 with the radius equated at height, $h$, on the primary spin axis with - denoting the high pressure plate (B), and + denoting the low pressure plate $(\mathrm{A})$ :

$U_{T+}=U_{\infty}+r_{h} \omega$

$U_{T-}=U_{\infty}-r_{h} \omega$

The skin friction coefficient $\mathrm{C}_{\mathrm{F}}$ is also calculated separately for the high and the low pressure plate using the standard flat plate model and is given by:

$C_{F \pm}=0.0074\left(\frac{U_{T \pm} \rho d}{\eta}\right)^{-\frac{1}{5}}$

Substituting into equation 8 , the total force is given in equation 9:

$F_{T}=\rho \pi r^{2}\left[U_{T+}^{2} C_{F+}+U_{T-}^{2} C_{F-}\right]$ 
Consequently the angular deceleration of the ball due to surface friction, with the total torque estimated to act at the centre of the plate, is given by:

$$
\dot{\omega}=\frac{T}{I} \text { Where } T=F_{T} r / 2
$$

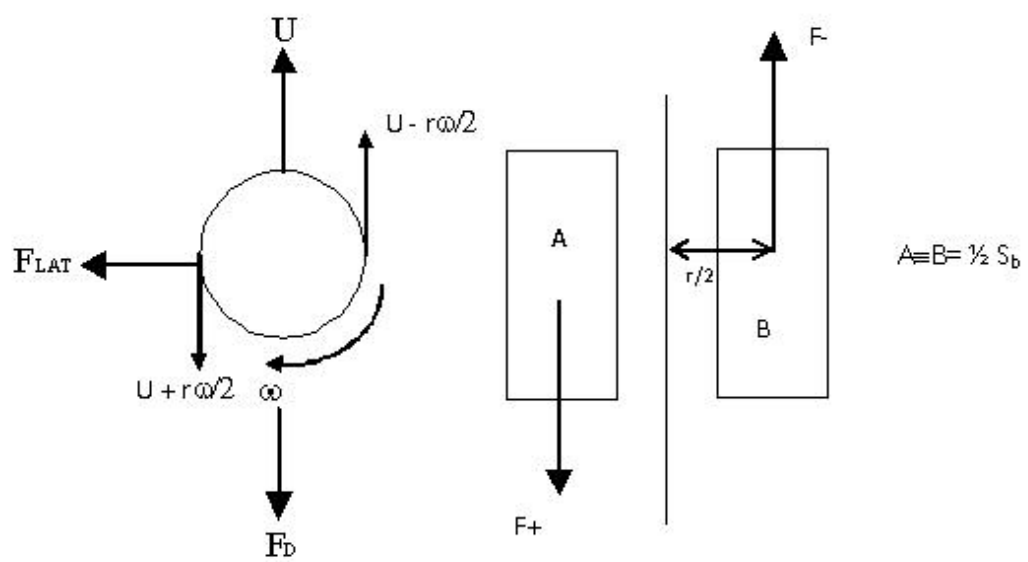

Figure 5 Ball equated as two flat plates

\section{Experimental Methods}

To generate the empirical model of spin rate decay a new wind tunnel test was developed to measure the coast-down behaviour of the ball and extract the spin decay parameters. The data was acquired in the low speed, open circuit, closed jet wind tunnel at Loughborough University. Detailed information regarding the design and performance of the tunnel can be found in Johl ${ }^{17}$ In this tunnel a standard size 5 football produces a blockage ratio of only $1.70 \%$ thus the data has not been corrected for blockage. The tunnel is capable of achieving an upper Reynolds numbers of $\mathrm{Re}=7 \times 10^{5}$ based on a football diameter of $0.220 \mathrm{~m}$.

The ball, located in the wind tunnel working section, was supported from below, mounted on an ultra-low friction $(\mu<0.002)$ air bearing (loaned from Air Bearings Ltd, UK) located under the tunnel floor. The ultra-low friction set-up makes it possible to separate the ball spin decay from the mechanical losses. With the tunnel speed held constant $(0,10,2030, \mathrm{~m} / \mathrm{s})$, the ball was accelerated to $52.36 \mathrm{rad} / \mathrm{s}(500 \mathrm{rpm})$ and then allowed to decelerate freely. The spin rate was determined using an optical taco with 6 reflectors; the signal was processed through a frequency to voltage convertor and sampled at $625 \mathrm{~Hz}$.

The resistive torque generated both from the surface friction and the moment arising out of the lateral force $T\left(\omega, M_{\psi}\right)$ was extracted from the coast-down data using a non-linear optimisation routine. The equation of motion is given by equation 13 .

$T\left(\omega, M_{\psi}\right)=I_{e} \frac{d \omega}{d t}$ 
Differentiating the spin rate will introduce noise to the signal, thus it is more accurate to calculate the torque using the integral of equation 13 . Hence by rearrangement:

$\omega=\int_{t_{0}}^{t_{1}}-\frac{T}{I} d t$

The spin rate against time curves are filtered using a low pass frequency filter, with a cut off frequency of $1 \mathrm{~Hz}$, to remove ripple associated with the frequency to voltage converter and any other noise sources. The friction component is approximated by a quadratic and the coefficients of the quadratic obtained using a Nelder-Mead ${ }^{18}$ non-linear optimisation within Matlab ${ }^{\mathrm{TM}}$. To separate the skin friction from the aerodynamic yaw moment the moment is determined separately from the standard wind tunnel test data. The cost is calculated from a comparison between equation 14 and the measured spin rate data to form a sum of errors squared.

$$
F(\mathbf{x})=\sum_{i=1}^{m}\left[f_{i}(\mathbf{x})\right]^{2}=\sum_{i=1}^{m}\left[\omega_{m_{i}}-\omega_{i}(\mathbf{x})\right]^{2}
$$

By varying the coefficients in the vector $\mathrm{x}$ the optimisation routine converges to a minimum of the cost to give the best estimate of the coefficients. The resulting function thus equates directly to the surface friction.

\section{Results}

\section{Spin Rate Degradation}

Figure 6 shows the raw spin rate decay data for a standard 32 panel football. As the velocity increases there is an increased initial rate of decay, but at the higher velocities this decay rate significantly reduces with time and at $30 \mathrm{~ms}^{-1}$ levels off to $11.5 \mathrm{rad} / \mathrm{s}$.

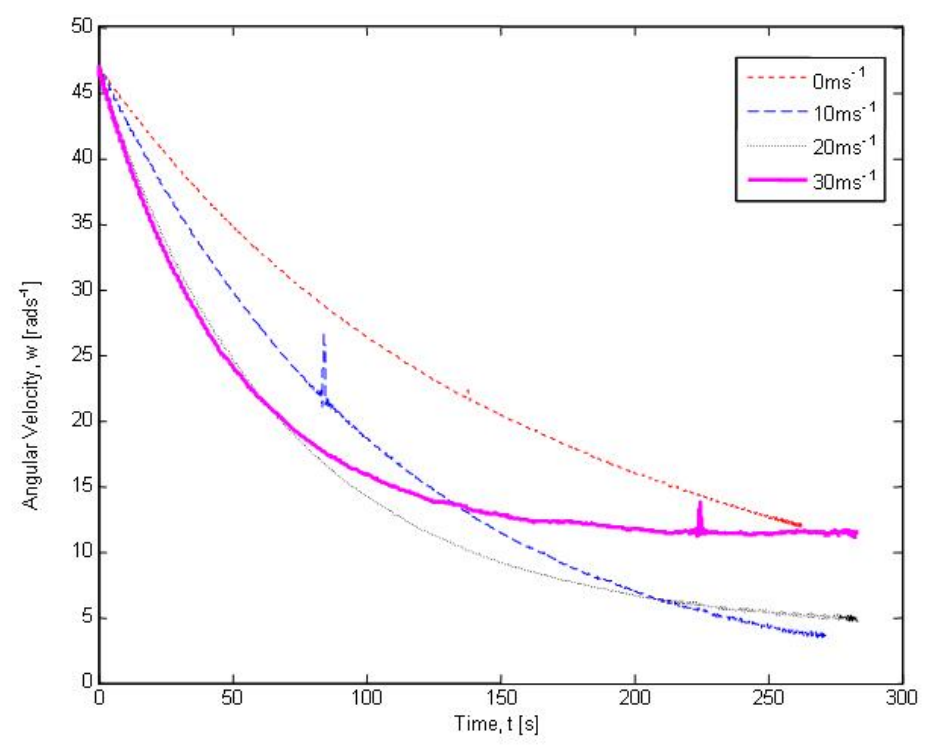

Figure 6 Spin Rate Decay of a 32 Panel Football 
The lateral deviation after $25 \mathrm{~m}$ of free flight with varying initial spin rates for a kick launched at $30 \mathrm{~m} / \mathrm{s}$ and $18^{\circ}$ elevation is shown in Table 1 . Results are reported for each of the three approaches: no spin decay, simple model spin decay and measured spin decay.

\begin{tabular}{|cc|c|c|c|}
\hline \multicolumn{2}{|c|}{$\begin{array}{l}\text { Initial Spin Rate } \\
{[\mathbf{r a d} / \mathbf{s}]}\end{array}$} & $\begin{array}{c}\text { Lateral Deviation [m] } \\
\text { [Rpm] }\end{array}$ & $\begin{array}{c}\text { Lateral Deviation [m] } \\
\text { Simple model of SRD }\end{array}$ & $\begin{array}{c}\text { Lateral Deviation [m] } \\
\text { No SRD }\end{array}$ \\
\hline 10.47 & 100 & 1.71 & 1.94 & 1.96 \\
\hline 20.94 & 200 & 2.57 & 2.71 & 2.75 \\
\hline 31.42 & 300 & 3.14 & 3.25 & 3.29 \\
\hline 41.89 & 400 & 3.65 & 3.74 & 3.80 \\
\hline 52.36 & 500 & 4.12 & 4.19 & 4.26 \\
\hline
\end{tabular}

Table 1 Consequence of Spin Rate Decay on Lateral Deviation after 25m of Free Flight

In comparison to applying the assumption of a constant spin rate, the two methods of implementing the decay in the flight model result in a reduction in the lateral deviation, with the simple model producing significantly less overall deviation. For example at an initial spin rate of $31.4 \mathrm{rad} / \mathrm{s}(300 \mathrm{rpm})$ the simple model indicates a reduction in lateral deviation of $0.15 \mathrm{~m}$ or approximately 0.7 ball diameters whereas the spin rate decay calculated from the experimental data results in a reduction of only $0.04 \mathrm{~m}$, less than 0.2 ball diameters.

\section{Influence of the drag profile}

The effect that the drag coefficient against Reynolds number (drag profile) has on the practical flight of the ball can be explored within the flight model by using idealised drag profiles. The idealised data used is shown in the four plots in figure $7 \mathrm{a}$, this simulated data allows the effects of transition Reynolds number, the rate of transition and the level of post transition $C_{D}$ each to be studied in isolation. The data (figure $7 \mathrm{a}$ ) is representative of the characteristics seen in typical real balls (see figure 1). Of immediate interest when undertaking this was that it became clear that in a game situation it is difficult to get the flight from a realistic kick to pass through transition (through velocity degradation) before the ball strikes the ground, and further that the forces present below transition are sufficiently small that they have little effect on the flight. Thus to investigate the effects of transition point (CD1-CD3 figure 7a) and transition slope (CD4-CD9 figure 7a) the launch conditions were chosen such that the flights are forced to go through transition, this required somewhat contrived initial conditions of velocity at $18 \mathrm{~m} / \mathrm{s}$ and a launch angle of $30^{\circ}$ (Launch Condition 1). To show the effect of post transition $C_{D}$ (CD10CD14 figure 7a) a launch condition of $30 \mathrm{~m} / \mathrm{s}$ at $18^{\circ}$ (Launch Condition 2) was selected to ensure that the ball velocity did not reduce below the transition point. Figure $7 \mathrm{~b}$ demonstrates typical x-z flight trajectories: 

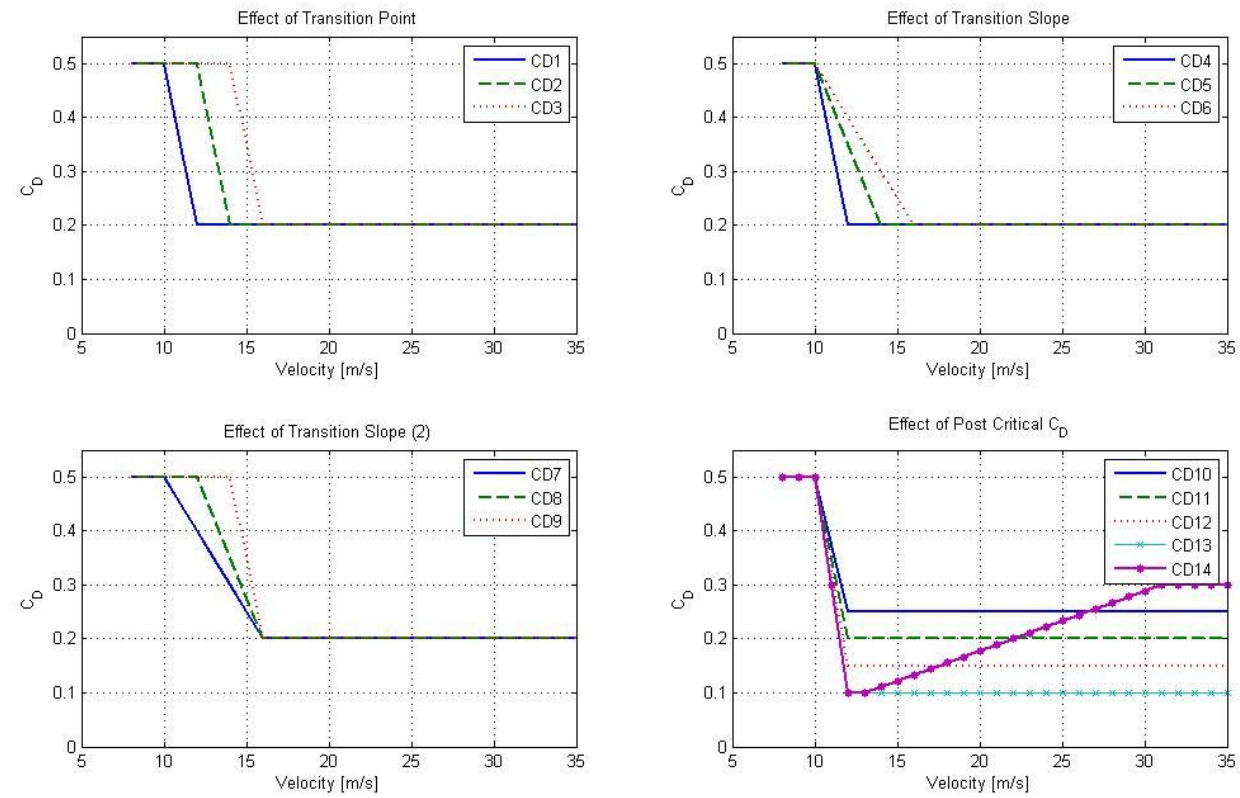

Figure 7a Simulated Reynolds Sweep Data

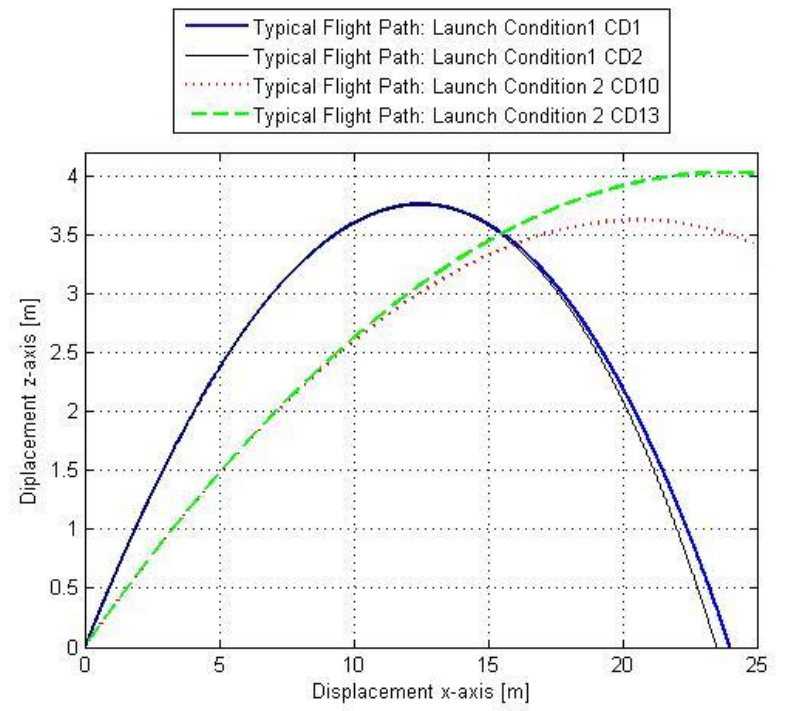

Figure 7b Typical Flight Trajectories for Simulated Reynolds Sweep Data 
Tables $2 \mathrm{a}$ and $2 \mathrm{~b}$ summarise the influence of Reynolds data by presenting the total velocity of the ball after the complete free flight period for each of the Reynolds sweeps presented in figure $7 \mathrm{a}$.

\begin{tabular}{|c|c|c|c|}
\hline Reynolds Data & $\begin{array}{c}\text { Velocity @ Ground } \\
\left.\text { Strike [ } \mathbf{m s}^{-\mathbf{1}}\right]\end{array}$ & Reynolds Data & $\begin{array}{c}\text { Velocity @ Ground } \\
\text { Strike [ms }\end{array}$ \\
\hline CD1 $]$
\end{tabular}

Table 2a Summary of Reynolds Experiment: Launch Condition 1: Initial velocity of $18 \mathrm{~m} / \mathrm{s}$ at a launch angle of $30^{\circ}$

\begin{tabular}{|c|c|}
\hline Reynolds Data & $\begin{array}{c}\text { Velocity @ Ground } \\
\text { Strike }\left[\mathbf{m s}^{-\mathbf{1}} \text { ] }\right.\end{array}$ \\
\hline CD10 & 19.3 \\
\hline CD11 & 20.6 \\
\hline CD12 & 22.2 \\
\hline CD13 & 24.2 \\
\hline CD14 & 20.5 \\
\hline
\end{tabular}

Table 2 b Summary of Reynolds Experiment: Launch Condition 2: Initial velocity of $30 \mathrm{~m} / \mathrm{s}$ at a launch angle of $18^{\circ}$

\section{Discussion}

\section{Spin Rate Decay}

Examining the raw coast-down curves (figure 6) reveals that as the airspeed increases there is an increased initial rate of decay, but at the higher wind speeds this decay rate reduces significantly as the angular velocity reduces. This is particularly pronounced at $30 \mathrm{~ms}^{-1}$ where the angular velocity levels off to $11.5 \mathrm{rad} / \mathrm{s}$. This is a consequence of the yaw moment becoming more dominant with the offset centre of pressure invoking a positive torque on the ball to counteract the skin friction and maintain the spin rate. Consequently it is imperative to include the yaw moment term (equation 7) within the flight model if spin rate decay is to be included in the form of a simple model. If the experimentally based data is used directly then this term is not required as the effect can be contained within the spin rate decay function.

Dimensionless analysis of the model shows that for the spin rate decay $\left(\dot{\omega} r^{2} / U_{\infty}^{2}\right)$ all results collapse onto a single curve for all Reynolds numbers; this is in agreement with Smits ${ }^{19}$ who obtained similar results for a golf ball. This implies that, as stated in Smits ${ }^{19}$, that spin rate will decay exponentially with time for a constant velocity, or exponentially with distance if velocity varies. Thus spin rate decay is proportional to the initial spin rate and launch velocity. This effect is seen within the experimental data and suggests that the model has the correct approach. As seen in Table 1, the effect of including experimentally determined spin rate decay is a small reduction in lateral deviation. Alternatively, using the simple model overestimates the reduction in spin rate giving less lateral deviation for the same conditions. This result shows that in this 
case implementing an inaccurate model is potentially worse than not using one at all. A more sophisticated model could be developed by integrating an estimate of the local skin friction coefficient over the ball, but would also require knowledge of the actual separation line. This is difficult and time consuming to perform, potentially requiring a full CFD simulation, and would negate the value of a simple approach. The alternative is to take the experimental approach used here, because while a single ball has been tested and reported here, it is possible to identify the different spin degradations for any ball type and thereby make comparisons. Even a relatively sophisticated model is unlikely to be sufficiently accurate to achieve this.

For the purposes of generating representative flights and making comparisons between balls the small change in lateral deviation that the spin degradation gives rise to does not warrant its inclusion in the flight model. However if the purpose is to replicate an actual real world kick then it should be included.

\section{Influence of the drag profile}

The data presented in tables $2 \mathrm{a}$ and $2 \mathrm{~b}$ suggest a broad dependency on the mean $\mathrm{C}_{\mathrm{D}}$ rather than the complete drag profile. The influence of the point of transition and shape of the Reynolds response (CD1-CD9) shows some variation in the final velocity, however, with these initial launch conditions the flight time for the ball to travel $25 \mathrm{~m}$ varies by only $\pm 50 \mathrm{~ms}$, suggesting there will be little difference in the perception of the flight from a player perspective. The significance of these differences is further reduced by the fact that the player does not have exact knowledge of the initial velocity of the ball and naturally adjusts their position to receive throughout the flight. It is also worth recalling that in order to generate the data it was necessary to somewhat contrive the kick. The post critical $C_{D}(C D 10-C D 14)$ uses a more realistic free kick $^{6}$ and has a much larger effect on the flight; it is therefore potentially a more influential parameter in a practical game situation.

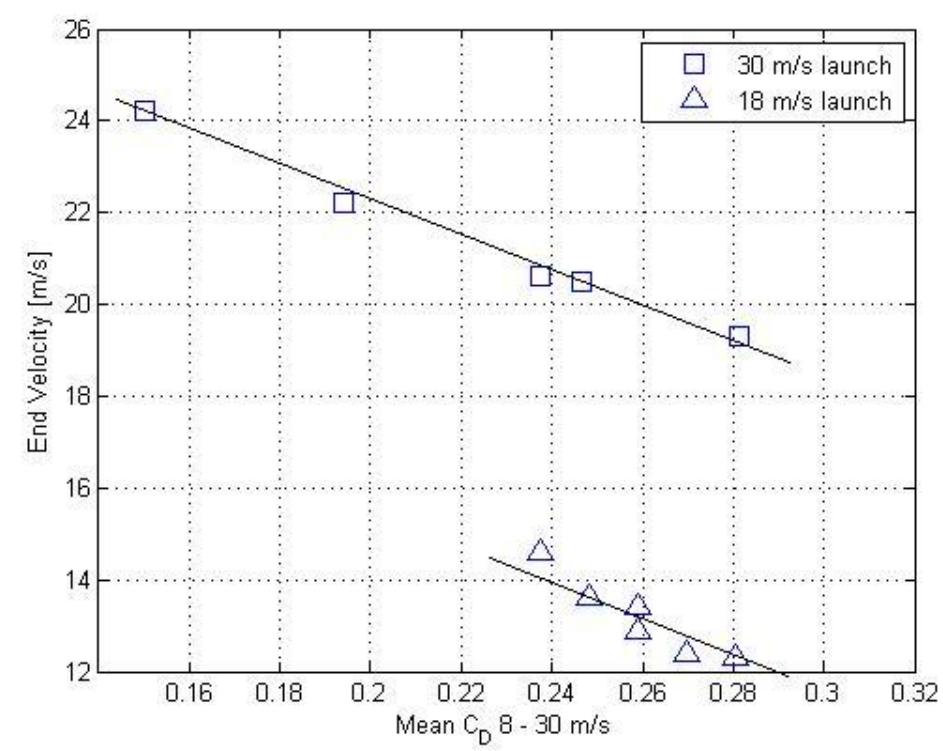

Figure 8 Summary of Reynolds Behaviour 
Plotting end velocity for all Reynolds characteristics in figure 8 against the mean $C_{D}$ (mean taken between 8 and $30 \mathrm{~ms}^{-1}$ of the drag profile) collapses the data onto two lines representing the two initial launch conditions. This demonstrates that the end velocity of a flight is proportional to the mean $C_{D}$ during the flight and thus will determine the total flight time. It may therefore be more appropriate to use an average value of the drag coefficient in the flight model or, given that the ball will spend much of its flight time in the post transition condition for the majority of kicks, an average of the post critical values. Finally it should be recognised that this analysis is limited to the influence of the drag coefficient and that the analysis and consequent conclusions are likely to be different for the lateral effects.

\section{Conclusions}

The use of a flight model based on wind tunnel measured aerodynamic data has been shown to be a useful tool for predicting ball performance and for investigating a number of aspects of ball flight. A simple skin friction based model of spin rate decay is compared with experimentally derived data and the simple model is shown to overestimate the spin rate decay. A more sophisticated model could be developed by integrating an estimate of the local skin friction coefficient over the ball, but is a complex and time consuming task. The preferred method is therefore to use the experimental approach used here; this has the advantage that it is then also possible to identify the different spin degradations for any ball type The inclusion of spin rate decay in the flight model compared to a constant spin rate is shown to cause a small reduction in the overall lateral deviation of the ball in a typical kick. It may be unnecessary to include this when making comparisons between ball types but would be an important consideration when attempting to correlate between simulated and measured real flights.

The shape of the drag coefficient against Reynolds number (drag profile) response is explored with regard to critical Reynolds Number, the rate of transition and level of post transition $C_{D}$ and it is shown that it is unlikely to have an impact on the player perception of flight. In this regard it may be sufficient to include only a mean value of $C_{D}$ in a flight model or mean post critical drag coefficient. In the simulation of the effects of Reynolds number the analysis shows that for realistic Reynolds characteristics there are few game scenarios in which the ball passes through transition.

\section{References}

1. Alam, F., Chowdhury, H., et al. A comparative study of football aerodynamics. $8^{\text {th }}$ Conference of the ISEA, Procedia Engineering V 22443 - 24482010.

2. Achenbach, E., The effects of surface roughness and tunnel blockage on the flow past spheres, Journal of Fluid Mechanics, V65(1) pp113-125, 1974

3. Achenbach, E., Experiments on the flow past spheres at very high Reynolds numbers, Journal of Fluid Mechanics, V54(3) pp565-575, 1972

4. Achenbach, E., Vortex Shedding from Spheres, Journal of Fluid Mechanics, V62 (2) pp209-221, 1974.

5. Mehta, R.D., Aerodynamics of Sports Balls, Ann. Rev. Fluid Mech. Annual Reviews inc. 1985

6. Passmore, M.; Rogers, D.; Tuplin. S.; et al. The aerodynamic performance of a range of FIFA approved footballs. Proc. IMechE Part P J. Sports Engineering and Technology, 2011 
7. Passmore, M.; Spencer, A.; Tuplin, S.; Jones, R. Experimental studies of the aerodynamics of spinning and stationary footballs. Proc. IMechE Part C J. Mechanical Engineering Science, Volume 222 (C2), pp 207-211, 2008

8. Asai, T., Seo, K., Kobayashi, O., Sakashita, R., Fundamental aerodynamics of the soccer ball, Sports Engineering, V(10) 101-110. 2007.

9. Carre, M.J., Goodwill, S.R., Haake, S.J., Understanding the effect of seams on the aerodynamics of an association football. Proc. ImechE Part C Mechanical Engineering Science V(219) pp 657- 6662005.

10. Bray K, Kerwin D. G, Modelling the flight of a soccer ball in a direct free kick. Journal of Sports Sciences, V 21, pp 75-85, 2003.

11. Goff, J.E., Carre, M.J., Trajectory analysis of a soccer ball. Am. J. Phys. V 77(11) pp 1020 - 10272009

12. Goff, J.E., Carre, M.J., Soccer ball lift coefficients via trajectory analysis. Eur J. Phys. V 31 pp $775-784,2010$

13. Cook, B.G., Goff, J.E., Parameter space for successful soccer kicks. Eur. J. Phys. V27 pp 856-874. 2006.

14. James, D., Haake, S., The spin decay of sports balls in flight. The Engineering of Sport $7-V 2$ P172. 2008

15. Price, D.S. Advanced Modeling of Soccer Balls. PhD Thesis, Loughborough University, Loughborough UK. 2005.

16. Neilson, P.J., The Dynamic Testing of Soccer Balls, Doctoral Thesis, Loughborough University, 2003.

17. Johl, G., Passmore, M.A., Render, P., The Design Methodology and Performance of an Indraft Wind Tunnel, The Aeronautical Journal, V108 (1087), pp. 465-473. 2004.

18. Nelder, A.J., Mead, R., 'A Simplex Method for Function Minimization' The Computer Journal, Volume 7, Issue 4, 1965

19. Smits, A.J., A New Aerodynamic Model of a Golf Ball in Flight. Science \& Golf II Proceedings of the World Scientific Congress of Golf, E \& FN Spon, London, 1994. 